\title{
Control Concepts for Facilitating Motorway On-ramp Merging Using Intelligent Vehicles
}

\author{
RICCARDO SCARINCI* AND BENJAMIN HEYDECKER \\ Department of Civil, Environmental and Geomatic Engineering, Centre for Transport Studies, \\ University College London, Gower Street, WC1E 6BT London, UK
}

(Received 21 March 2014; revised 20 October 2014; accepted 29 October 2014)

\begin{abstract}
Congestion at motorway junctions is a traffic phenomenon that degrades operation of infrastructure and can lead to breakdown of traffic flow and associated reduction in capacity. Advanced communication technologies open new possibilities to prevent or at least delay this phenomenon, and innovative active traffic management systems have been developed in the recent years for better control of motorway traffic. This paper presents a review of control strategies for facilitating motorway on-ramp merging using intelligent vehicles. First, the concepts of the control algorithms are reviewed chronologically divided into three types of intelligent vehicle: completely automated, equipped with cooperative adaptive cruise control and equipped with on-board display. Then, a common structure is identified, and the algorithms are presented based on their characteristics in order to identify similarities, dissimilarities, trends and possible future research directions. Finally, using a similar approach, a review of the methods used to evaluate these control strategies identifies important aspects that should be considered by further research on this topic.
\end{abstract}

\section{Introduction}

Congestion on motorways (USA: freeways) is a phenomenon affecting society, the economy and the environment. It often takes place at specific locations such as onramps, lane drops, crests, sags and sharp bends. Particularly recurrent and degrading for the infrastructure is congestion at on-ramps, which can cause a drop in capacity and blockages of upstream off-ramps (Bertini \& Malik, 2004; Leclercq, Laval, \& Chiabaut, 2011; Yi \& Mulinazzi, 2007). Research on the management and avoidance of this phenomenon has been carried out since the development of motorway roads. Initial attempts to decrease and prevent congestion at merges were related to the physical improvement of the infrastructure layout, trying to identify the optimal junction design (Fukutome \& Moskowitz, 1960; Harwood \& Mason, 1993; Hunter, Machemehl, \& Tsyganov, 2001). Subsequently, the attention moved to active traffic management (ATM) strategies, and systems such as ramp metering have been introduced to control on-ramp flows and

*Corresponding author. Email: riccardo.scarinci.09@ucl.ac.uk 
avoid or at least delay breakdown on the main carriageway (Papageorgiou \& Kotsialos, 2002). Nowadays, the research focuses on innovative algorithms in ATM for optimising motorway merging, which is reviewed in the present paper. These new control strategies are based on emerging vehicle-to-vehicle $(\mathrm{V} 2 \mathrm{~V})$ and vehicle-to-infrastructure (V2I) communication technologies, more accurate positioning systems, in-car driver assistance devices and on a better understanding of traffic flow phenomena. These advances open new possibilities in the field of ATM, and particularly promising is the cooperation among vehicles enabled by the V2V and V2I communication. This new research thread is known as cooperative intelligent transport system (CITS) (NEARCTIS, 2009). Since the early 1990s, a multitude of innovative algorithms have been presented and evaluated but, unlike other ATM areas, no review paper has been published with focus on control at motorway merges.

The present work reviews control strategies for facilitating motorway on-ramp merging using intelligent vehicles. A common structure is identified, and the algorithms are presented based on their characteristics together with the methodologies used for evaluating their quality. Finally, similarities and differences among the control strategies are summarised as well as research trends and possible directions for future research.

This paper reviews algorithms for motorway on-ramp merging intended to assist the merging process using emerging technologies. To better understand the scope of the review, it is convenient to identify some examples of related research topics that are not included. The ramp metering system, even if it uses intelligent vehicles to estimate the traffic state, is not included because the control strategies aim to prevent the congestion but do not intend to facilitate the merging process (Kerner, 2007; Masher, 1975; Papageorgiou, Hadj-Salem, \& Blosseville, 1991). Algorithms for the automatic control of platoon formation on the main carriageway are not included for the same reason (Shladover, 1991; Swaroop \& Hedrick, 1999). Similar consideration applies to algorithms using adaptive cruise control with the exclusive aim of increasing string stability (van Arem, van Driel, \& Visser, 2006; Darbha \& Rajagopal, 1999). Also, systems related to the concept of "infrastructure-assisted merging" are not reviewed. An example of this type of systems is the use of lights positioned alongside the on-ramp which, sequentially illuminated, suggest the merging speed to drivers (Tignor, 1975). Finally, the technological requirements are not considered explicitly. Although the paper presents the type of intelligent vehicles and the communication needed, it introduces the control strategies independently from their technological requirements.

The paper is organised as follows: the next section describes the components involved in the merging process of intelligent vehicles. Then, a chronological review of the control strategy is given, followed by an overview where similarities and differences are emphasised for both control strategies and evaluation methods. The paper finishes with the main conclusions.

\section{Characteristics of the Controlled Merging Process}

In this section the infrastructure, the vehicles, the technologies and the algorithms involved in the controlled merging process are introduced. In order to have a common terminology for describing the various control strategies suitable for the scope of this overview, the components are here defined and represented graphically in Figure 1 . The resulting definitions are an abstraction and 


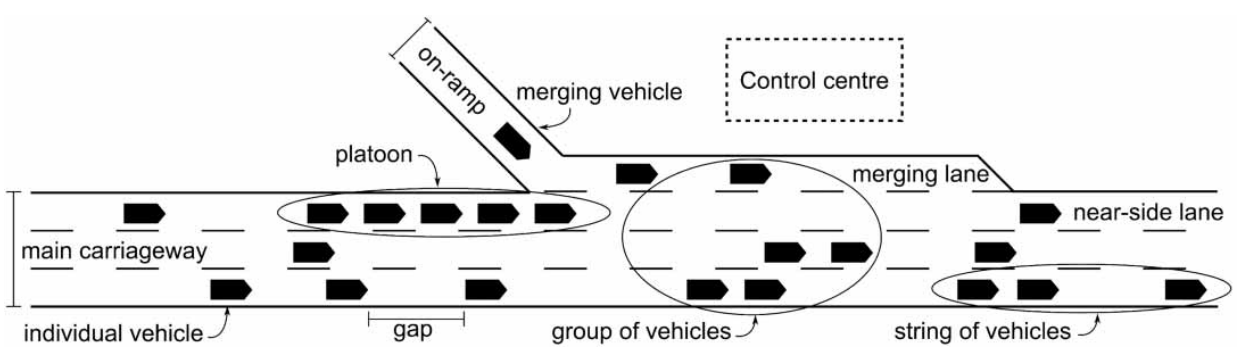

Figure 1. Graphical representation of the components involved in the merging process of controlled intelligent vehicles.

summary based on the different terms used by various authors, and references to the specific papers can be found in Table 1.

The zone of motorway infrastructure where the merging occurs is composed of a main carriageway and an on-ramp. The main carriageway usually consists of multiple lanes where the most influential for the merging process is that closest to the on-ramp, here referred to as the near-side lane. The on-ramp is divided into a section completely detached from the main carriageway and a section where a lane change to the main carriageway is possible, which is known as the merging section (DfT, 2011).

Vehicles can move on the infrastructure in two directions: longitudinal and lateral. Longitudinal movements take place in the same lane, whereas lateral movements relate to lane-changing. In the case of controlled merging, both movements can be limited either by the traffic conditions or by the control algorithm. The merging manoeuvre is a special case of lateral movement, where an on-ramp vehicle moves from the merging lane to the near-side lane on the main carriageway.

Different vehicle formations can be identified. Consecutive vehicles travelling on the same lane are defined as a string of vehicles (Lu et al., 2004; Ran et al., 1999). A string of vehicles travelling with constant and small gaps between them are defined as a platoon, which can be either naturally formed or induced by the control algorithm. Finally, adjacent vehicles on multiple lanes are defined as a group (Kanavalli et al., 2008; Kato et al., 2002).

The vehicle composition can have different penetration rates, that is, percentage of intelligent vehicles over the total number of vehicles. Traffic can be completely formed by intelligent vehicles, that is, $100 \%$ penetration rate, or a mix of intelligent and normal vehicles, for example, $1 \%, 5 \%, 10 \%$, here referred to as mixed traffic. For the scope of this review, it is convenient to identify three different types of intelligent vehicle:

(1) Completely automated vehicles. These vehicles are fully autonomous and can perform longitudinal and lateral movements without human intervention summarised as 'hands-off' and 'feet-off'.

(2) Vehicles equipped with cooperative adaptive cruise control (CACC) (van Arem et al., 2006). CACC is an extension of adaptive cruise control (ACC), itself an extension of cruise control (CC). While vehicles equipped with $C C$ are capable of maintaining a fixed speed, vehicles equipped with ACC are capable of maintaining an appropriate, possibly speed-dependent, gap behind the preceding vehicle using forward-looking sensors. Finally, vehicles 
Table 1. Characteristics of merging control strategies

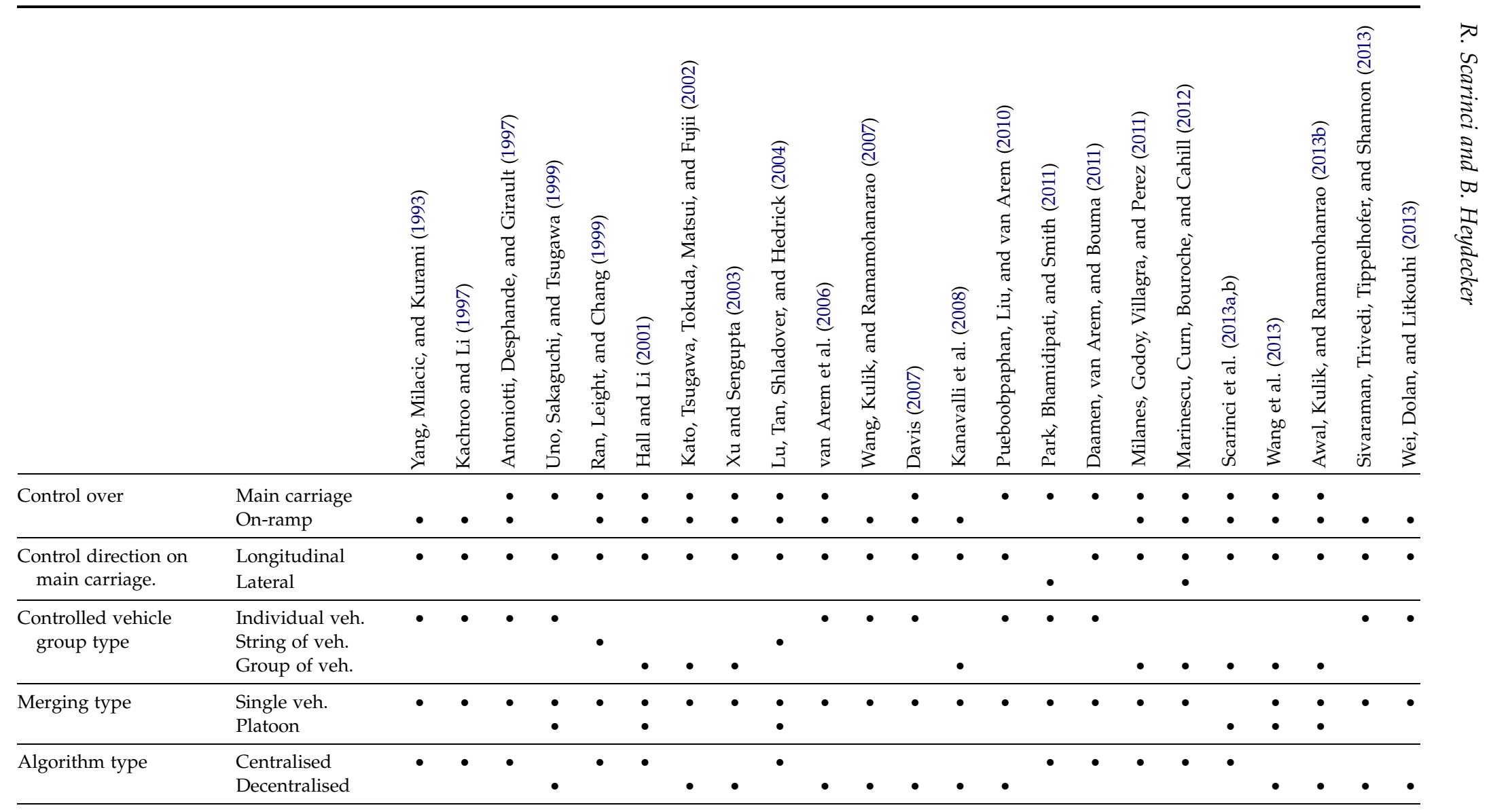




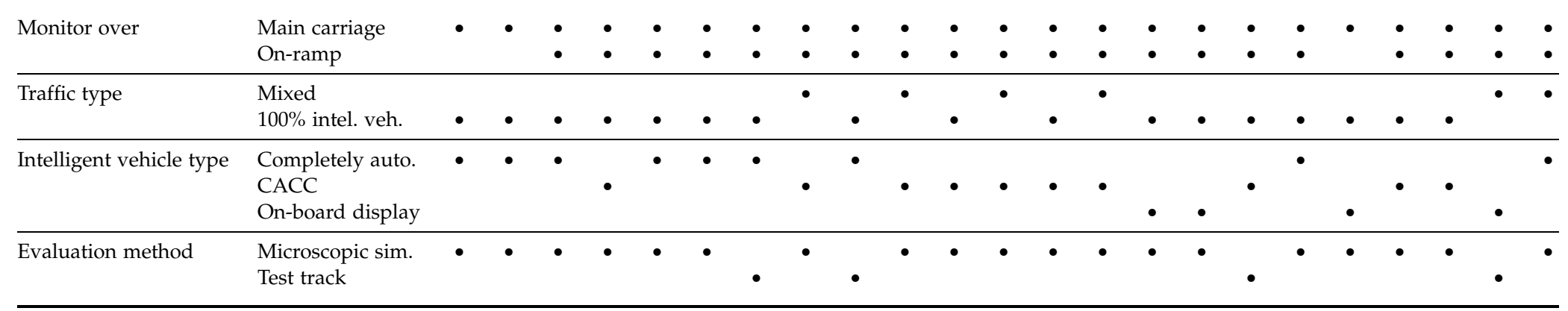


equipped with CACC are also capable of exchanging information with vehicles within their communication range and acting jointly with them.

(3) The third type of intelligent vehicles are vehicles equipped with an on-board display capable of receiving messages from the infrastructure containing advice to that the driver should consider.

All the three intelligent vehicle types are equipped with either or both of communication and control technologies able to receive information and to communicate position, speed and acceleration.

The last element of the merging process is the control algorithm. The algorithm can control intelligent vehicles on either or both of the main carriageway and the on-ramp. It can control (or advise the drivers, in the case of vehicles equipped with an on-board display) longitudinal or lateral movements. Longitudinal movements are controlled by modifying the vehicle speed whilst lateral movement by changing lane. The algorithm can monitor and control an individual vehicle, a string of vehicles or a group of vehicles either on the main carriageway or on the on-ramp. Finally, algorithms can be defined as centralised (junction level) or decentralised (vehicle level). Centralised algorithms collect information from the monitored vehicles in a single control centre for a specific junction and communicate instructions to the vehicles. On the other hand, in decentralised algorithms the 'intelligence' is distributed among vehicles, and no central control centre is required. This is usually the case of vehicles equipped with CACC, where each individual vehicle receives information from the vehicles within its communication range and calculates its own control action.

\section{Review of Merging Control Strategies}

This section presents a chronological review of the control strategies developed since the topic of merging assistance gained the attention of researchers. Because algorithms adopting the same type of intelligent vehicle present similarities, the review is divided into three sections, one for each technology type used: completely automated vehicles, vehicles equipped with CACC and vehicles equipped with on-board display. The review presents the concepts of the control strategy rather than the details of the specific algorithms and the methodology used to design and optimise the control law, which can be found in the original papers. Here only the review is reported, and the summary and discussion of the controls presented are carried out in the following sections.

\subsection{Completely Automated Vehicles}

Initial studies on automated merging manoeuvres can be linked to a specific type of urban public transport system, the automated guideway transit (AGT), a driverless train operating on exclusive guideways (Athans, 1969; Sarachik \& Chu, 1975; Shladover, 1980). Although these studies implemented different merging configurations, including strategies for platoon merging, the dynamics for trainlike vehicles are different from the ones for car-like vehicles; therefore, this research will be not reviewed here.

Early work on completely automated intelligent vehicles focused mainly on longitudinal behaviour, such as platoon formation, rather than on facilitating the merge itself. The first research on controlled merging was developed within 
the Intelligent Vehicle Highway Systems programme in the early 1990s (Mammano \& Bishop, 1992; Varaiya, 1993). As part of this USA programme, the Federal Highway Administration began to study an automated highway system (AHS), which was described as "a system of instrumented vehicles and highways that provides fully automated (i.e. 'hands-off') operation, improving safety, efficiency and comfort" (Bishop \& Stevens, 1993, p. 365). As a result of this project, several papers were published on control algorithms for facilitating the merging manoeuvre using completely automated vehicles. In the following algorithms, if not stated otherwise, the vehicle positions, speeds, accelerations and intervehicle gaps are identified from V2I and V2V communication for centralised and decentralised systems, respectively.

Yang et al. (1993) and Yang and Kurami (1993), within the AHS programme, presented a control algorithm that had the aim of guiding on-ramp vehicles into gaps on the main carriageway, and so to provide smooth merging. The control regulates the speed profile of the merging vehicle based on its position relative to the target gap. The formation or preservation of main carriageway gaps is not part of the algorithm. The on-ramp vehicle speed is defined by a guidance law divided into two phases. The first one, 'long-range soft homing', controls the vehicle speed when there is a long distance to the gap. The second phase, 'short-range hard pushing', controls the trajectory in the final section of the gap alignment. A speed regulator controls the vehicle throttle and brake position in order to maintain the required speed. A feedback system is used to define the two-phase guidance law. Computer simulation, reporting gap and speed profiles of individual vehicles, shows that smooth merging can be achieved using this control strategy.

Subsequently, Kachroo and Li (1997), also within the AHS programme, proposed three new guidance laws for defining the speed profile of merging vehicles into gaps naturally present on the main carriageway. Similar to Yang et al., the formation and preservation of gaps in the main carriageway are not controlled. The three guidance laws (linear, optimal and parabolic) had increasing complexity in the speed profile of the merging vehicle, and consider the case of merging vehicles coming to a complete stop on the on-ramp in the absence of suitable gaps in the main carriageway flow. The control of the merging vehicle involves two feedback loops. The outer defines the desired behaviour of the on-ramp vehicle, while the inner constantly recalculates the final inputs based on the actual vehicle state. Methodology from calculus of variation was used to design the optimal strategy, and the vehicle merging control system was considered as a class of simple isoperimetric problem. Gap and speed profiles, obtained from microscopic simulation, showed excellent merging performance.

Antoniotti et al. (1997), differently from Yang et al. and Kachroo and Li, reported an algorithm that controls both on-ramp and main carriageway vehicles. The algorithm, once again developed within the AHS programme, has the objective of avoiding collisions, ensuring merging and maintaining the desired headway and normal speed. The speed of on-ramp vehicles is modified in two phases: 'align to gap', where the merging vehicle seeks a gap and attempts to align itself, and 'merge', when the lane change happens. The speed of main carriageway vehicles is modified during two phases of 'cruise' and 'yield'. In the 'cruise' phase, the speed is kept constant, while in the 'yield' phase vehicles increase the headway until a gap suitable for merging is created. The controller is designed based on a series of logical behavioural steps, and the acceleration profiles are a 
function of the headway and relative speed. Speed profile, gap profile and queue length have been evaluated with microscopic simulation. Results show that for low flows, the demand can be supported without queue build-up, whereas for high flows, the demand cannot be supported without a queue formation.

Two years later, and still part of the AHS programme, Ran et al. (1999) evaluated the traffic performance of a new algorithm for full automated merging similar to the one presented by Antoniotti et al. but with control over a string of vehicles rather than a single vehicle. The control aims to match merging vehicles to gaps specifically created on the main carriageway. The speed profile of on-ramp vehicles, including the possibility of stopping on the on-ramp, is controlled by specific sub-model: 'platoon following', 'gap checking and adjustment' and 'deceleration for metering'. Vehicles on the main carriageway can create gaps and consolidate them creating a platoon using completely automated vehicle trajectories defined by 'platoon forming' and 'intra-platoon following' models. The core of the system architecture of the merging control is the processor cell. The processor simulates the control actions based on input such as vehicle positions and speeds, and then a post processor generates the results. The authors focused on the evaluation of the traffic performance in different merging scenarios, and a microscopic simulation model has been developed incorporating the automatic merging control strategy. Results demonstrate that the algorithm can postpone the start of breakdown even when flow is close to capacity.

Hall and Li (2001), in the research thread of AHS, studied a merging strategy considering specific rules for different vehicle types, that is, passenger vehicles and heavy goods vehicles. The authors noticed that along with the vehicle characteristics such as acceleration and length, the merging behaviour also depends on the type of vehicles. Furthermore, the merging behaviour is not only conditioned by the type of merging vehicle itself but also by the type of main carriageway vehicle in front of which the on-ramp vehicle will merge. Following this consideration, Hall and Li proposed to group vehicles of the same type on separate onramp lanes, and to release them according to the type of vehicles on the main carriageway. The algorithm for fully automated vehicles looks for suitable gaps on the main carriageway and, proportionally to their size, releases an appropriate number of on-ramp vehicles in a platoon formation. The performance of different release strategies was evaluated using a microscopic simulation model calculating traffic indexes such as average waiting time in merging queue, average merging queue length and capacity. Results showed that the motorway throughput is maximum when on-ramp vehicles merge in front of main carriageway vehicles of the same type.

In 2002 Kato et al. reported the results of a field test of cooperative driving with automated vehicles. Using inter-vehicle (V2V) communication, the vehicles are formed into a platoon, in the case of a single-lane motorway, or into a grid, in the case of multi-lane, which facilitates smooth lane-changing and merging. Automated longitudinal and lateral algorithms manage the vehicle trajectories. Five automated vehicles have been tested on a test track considering different scenarios: stop and go, platooning, merging and obstacle detection. This demonstration showed the practicality and potential of the cooperative driving of automated vehicles but did not investigate the resulting traffic performance.

Afterward, Lu et al. (2004) also presented field test results of an algorithm for a fully automated merging manoeuvre as part of the AHS programme. If a convenient gap is already present on the main carriageway, the merging vehicle is 
guided to it; otherwise, the algorithm selects where to split the main carriageway platoon that is preventing the merge. Two vehicles on the main carriageway are separated to a prescribed safe distance and, once the gap is created, the algorithm generates a smooth reference trajectory for the merging vehicle. The authors suggested the possibility of merging a platoon of vehicles from the on-ramp instead of a single vehicle, simply considering multiple vehicles as an appropriate long abstract one. The controller used is the optimal dynamic backstepping sliding surface control (Lu, Tan, Shladover, \& Hedrick, 2001), which is a method that combines sliding mode, back-stepping and integral filters. The algorithm has been implemented and tested on a test track, and the practicality of the control strategy has been evaluated, but once again not the traffic performance.

Marinescu et al. (2012) proposed a merging algorithm using a 'slot-base' approach for completely automated vehicles. Each vehicle drives normally until the central traffic management system detects that the traffic conditions require a more efficient use of the infrastructure. At this point each vehicle is allocated to a virtual slot, and on-ramp vehicles are mapped into empty slots on the main carriageway that are selected by the central system for a smooth merging. The algorithm increases the merging possibility using the free slots on the other lanes of the motorway. A basic optimisation approach described in Marinescu, Curn, Slot, Bouroche, and Cahill (2010) is at the core of the control law. Microscopic simulation has been used to evaluate the algorithm performance, and results for medium and heavy traffic conditions show that this approach increases on-ramp throughput and decreases delay, thanks to the efficient merging process.

Finally, the important topic of interaction among autonomous and traditional vehicles is studied by Wei et al. (2013). The authors introduced a social behaviour framework for autonomous driving systems specific for on-ramp merging. During a transition period, manually driven vehicles will co-exist with automated vehicles, and the latter type should behave in such a way that human drivers can anticipate their movements in an intuitive way. For this reason, a more 'socially acceptable' motion planning was developed that takes into account the reactions of surrounding manually driven vehicles to the autonomous vehicle movements. The optimal merging trajectory of the automated vehicles is the one with the lower cost chosen among the possible ones. The cost is a combination of different functions designed to consider the desired distance, avoiding large accelerations, penalising small gaps and breaking distance and minimising fuel usage for all vehicles (whether autonomous or not) involved in the merge. The proposed algorithm was developed using a prediction and cost function approach that uses information based on the prediction of the movements of surrounding vehicles considering uncertainties in the sensor measurements. Simulation results show an increase in the smoothness of the speed adjustment and the maintenance of the safe distance. The occurrence of unsafe situations is reduced and the total cost decreased.

\subsection{Vehicles Equipped with CACC}

In a similar way to the completely automated vehicles, early work on CC, ACC and CACC concentrated more on the longitudinal behaviour of vehicles rather than facilitating the merging procedure. This research focused on evaluating the effects of the introduction of CC on safety, stability and capacity. 
Although not explicitly stated by the authors, the work of Uno et al. (1999) can be classified as one of the first research in the field of CACC for facilitating the merging process. Uno et al. proposed an algorithm based on the concept of 'virtual vehicle'. A virtual vehicle is mapped onto the main carriageway in order to control the creation of a gap. A main carriageway vehicle equipped with CACC will react to the presence of the virtual vehicle in front by decreasing its speed and increasing the headway. The gap in front that is created by this cooperative vehicle will then be used for a smooth merging. The algorithm maps the virtual vehicle in three different ways depending on how the merge is classified: at the beginning, at the end or in the middle of a main carriageway platoon. It is not completely clear whether or not the platoon recognition process necessary for the algorithm requires a centralised control, or the required information can be transmitted by inter-vehicle (V2V) communication, that is, decentralised control. The case of platoon merging is simply handled as a sequence of single vehicle merges. A microscopic simulation supports the practicality of the control algorithm, and speed profiles show smoother merging.

A few years later, $\mathrm{Xu}$ and Sengupta (2003) presented an evaluation of merging performance using vehicles equipped with CACC. Merging vehicles communicate in advance their intention of merging to intelligent main carriageway vehicles within their communication range. Receiving these messages, the main carriageway vehicles decrease their speeds in order to create suitable gaps. The desired acceleration is the control variable calculated using sliding surface, range error and the gain scheduling technique. Microscopic simulation presented encouraging results with an increase in the average speed and a decrease in braking efforts. Traffic performance was evaluated for different penetration rates of equipped vehicles (10-25-40-58-100\%), and it is shown that higher penetration rates are beneficial for the operation of this system. The control strategy aggressiveness is evaluated too. An aggressive control, that is, allowing stronger braking, increases the average speed, but a weaker control saves braking efforts, making the system safer and more comfortable.

Research on mixed traffic has received increasing attention, and in 2006 van Arem et al. evaluated the impact of CACC in the case of motorway lane merging due to a reduction in the number of lanes, which is known as lane drop. Although this paper does not evaluate merging from an on-ramp, it presents similarities to the present review scope. The authors demonstrated that CACC can have positive effects on traffic flow stability, thanks to the engaged cooperation among vehicles. The methodology used for the design of the controlled (speed and distance) can be found in VanderWerf, Shladover, Kourjanskaia, Miller, and Krishnan (2001). The results from a microscopic simulation for different penetration rates $(0-20-40-60-80-100 \%)$ and traffic flows close to capacity show promising results. Under the proposed control, the number of shock waves decreases drastically and the average speed increases. In contrast, the maximum observed traffic throughput shows small differences for different CACC penetration levels. Results also show that for penetration rates lower than $40 \%$, the impacts on traffic flow are small; instead, with penetration rates higher than $60 \%$, traffic stability is improved.

Opening a new research thread, Wang et al. (2007) presented and evaluated a range of merging algorithms for cars equipped with sensors capable of detecting and communicating position, speed and acceleration to the neighbour cars. This research field is defined as 'proactive merging strategy', but given the 
technological and algorithmic similarities with the field of CACC, this research can be inserted in the same thread. All the algorithms presented, that is, 'distance-based', 'speed-based', 'load-based', 'increase-based' and some combinations, aim to improve merging at motorway junctions. On-ramp vehicles choose specific gaps and, in preparation of merging, adjust their speed according to the gap position. The authors used a distance-based and speed-based algorithm to design the trajectories, where the main assumptions are that neither the speed nor the acceleration varies much, and the safety distance is guaranteed during the entire merging manoeuvre. Microscopic traffic simulation was used to evaluate the performance of the different algorithms in terms of delay, throughput, traffic flow and capacity. Their results showed improvements in performance for all the evaluated indexes, and a reduction in perturbations and sharp speed changes showed the increase in smoother merging.

Similar to $\mathrm{Xu}$ and Sengupta, and van Arem et al., Davis (2007) reported the traffic performance of a merging algorithm with mixed traffic flow consisting of vehicles equipped with CACC and manually driven. The objective of the merging algorithm is to create gaps on the main carriageway large enough that merging vehicles can change lanes without slowing down appreciably. Main carriageway vehicles adjust their speed and the relative position to the preceding vehicle prior to reaching the merging section. The trajectory of equipped vehicles is based on the generalised optimal velocity model, which is a string-stable control law that considers headway and extreme accelerations/decelerations. Microscopic simulation results for different penetration rates $(0-30-50-75-100 \%)$ show significant improvement in throughput and increase in distance travelled. With demand close to capacity, the algorithm was found to reduce congestion, but not entirely to suppress it. Once again, it was confirmed that the performance improves with the increase in the penetration rate of equipped vehicles.

Extending the research on 'proactive merging strategy' of Wang et al., Kanavalli et al. (2008) proposed an algorithm for merging of sensor-equipped vehicles. Cars are able to collect data related to vehicles in their surroundings and communicate to them. The decision to merge is taken ahead of the merging section, and the appropriate speed and acceleration are calculated when the vehicles are travelling on the on-ramp. The decentralised control is defined as a 'sliding-windows' merging algorithm, because it monitors the on-ramp and main carriageway vehicles inside a specific window whose size depends on the range of the sensors. The control law defines the optimal order of main carriageway and on-ramp vehicles for merging into a single stream. The algorithm identifies the configuration with minimal disturbance given the initial vehicle positions. Microscopic simulation was used to evaluate the traffic performance. Delay and throughput were improved by the use of the proactive algorithm even for high main carriageway flows.

Pueboobpaphan et al. (2010) considered a decentralised merging assistant for mixed traffic with the aim of increasing traffic flow stability by minimising conflicts in the merging section. Conflicts can be reduced encouraging early and smooth deceleration of main carriageway vehicles upstream of the merging area in order to create gaps for on-ramp vehicles. The merging assistant control law calculates the required acceleration/deceleration of equipped vehicles in order to minimise the number and magnitude of perturbations. Based on the predicted expected arrival time at merge location, the optimal change in speed is calculated and then compared with the standard comfortable rate for the 
driver. A microscopic traffic simulator has been used to evaluate the traffic performance for different main carriageway penetration rates $(0-50-100 \%)$, meanwhile all the on-ramp vehicles are driven manually. Total distance travelled, average travel time and number of collisions have been used as indexes of performance. Results show better performance in all the cases with CACC in comparison to manual traffic, although the merging assistant seems to be more effective under higher main carriageway demand and higher penetration rates.

Milanes et al. (2011) presented an advanced driver-assistance system controlling the longitudinal movement of both on-ramp and main carriageway vehicles. Although the algorithm is developed for urban environment, it is reviewed here because it presents many similarities with those developed specifically for motorways. The goal of the control strategy is to minimise the negative effects of the merging process on the main carriageway by providing a sufficient headway for smooth merging. The control architecture is divided into four steps: (i) perception - the controlled acquires the sensor measurements; (ii) management - the ways to solve the conflicts are identified; (iii) planning - the best alternative is chosen evaluating the traffic conditions and (iv) actuation - the control actions are executed. Fuzzy logic is used as a methodological approach for the problem-solving. The control strategy is evaluated with a field test using three equipped cars testing the system only at low speeds. Similar to the other field tests, no specific traffic indexes have been evaluated, but only the practicality of the algorithm.

With many similarities with the other algorithms in this group, Wang et al. (2013) evaluated an automated on-ramp merging strategy controlling the longitudinal movements of on-ramp and main carriageway vehicles, while the drivers are assumed to be capable of performing lane-changing. Using V2V communication, information, such as vehicle size, position and speed, is exchanged. The algorithm calculates, using kinematic equations, a smooth speed trajectory for the merging vehicles and the gap formation on the main carriageway. The algorithm aims to achieve the same speed for all vehicles involved in the merge, and to maintain the desired distance between these vehicles. Similar to Uno et al., to control the distances on the main carriage, a virtual vehicle is introduced by mapping the future position of the merging vehicle on the target lane. Simulation was used to evaluate the algorithm in two situations: (i) merging of a single on-ramp vehicle and (ii) merging of a platoon of on-ramp vehicles. The authors noted that the speed of upstream main carriageway vehicles is subject to stronger perturbations in the case of platoon merging. Unfortunately, no specific traffic indexes, for example, the number of shock waves or occurrence of break-down, are reported to quantify the performance of this approach.

Finally, using an optimisation approach, Awal et al. (2013b) proposed a proactive optimal merging strategy for merging two streams of intelligent vehicles. The optimal merging order of a group of vehicle is calculated based on knowledge of the positions and speeds of surrounding vehicles. Each vehicle in the group adjusts its speed to create an appropriate gap. The merging order is calculated using an iterative approach based on a recursive pruning algorithm. Merging time, waiting time, energy consumption, flow and average speed have been evaluated using a microscopic simulation model. The results showed improvement in all indexes, and in some scenarios, the proposed merging strategy achieves the best theoretical flow and marge rate. The only detrimental effect was that the average travel time on the main carriageway increased slightly. 


\subsection{Vehicles Equipped with On-board Display}

Considering vehicles equipped with on-board display for facilitating the merging process is a more recent research topic, and fewer studies have been carried out in comparison with the other two types of intelligent vehicles.

Park et al. (2011) developed an algorithm for advisory lane-changing intended to reduce merging conflict. Selected main carriageway vehicles are advised to change lane in order to create gaps for on-ramp vehicles. Firstly, the current position, speed and acceleration of vehicles in the merging area are collected, then possible necessary gaps are calculated and finally lane change advisory signals are shown to drivers. This algorithm calculates the expected gap sizes using kinematic equations and then selects the most appropriate lane-changing advice in order to maintain a minimum safety distance between vehicles. Microscopic simulation was used to identify the best advisory algorithm and to evaluate the traffic performance for different levels of drivers' compliance, that is, the proportion of drivers who follow the advice. Results showed an increase in average speed and a reduction in emission for compliance rates of $90 \%$ or higher. No significant changes in comparison with normal merging were observed for compliance lower than $50 \%$.

In the same period, Daamen et al. (2011) evaluated the possible improvements of sending messages to individual drivers in the case of the occurrence of two situations degrading for the infrastructure: large speed differences between vehicles on the same lane and platoons hindering merging vehicles at on-ramps. According to the scope of the present work, only the second situation is reviewed. The algorithm aims to improve the use of the motorway by showing messages on the on-board vehicle display. This approach has been defined as 'microscopic dynamic traffic management'. If a platoon of vehicles on the main carriageway is estimated to arrive at the merging area simultaneously with an on-ramp vehicle, a message is sent to a specific main carriageway vehicle requesting to decrease its speed to increase its headway, and so to create a gap for merging. Microscopic simulation results showed a significant improvement in the motorway throughput as well as a reduction in the travel time loss and in the number of shock waves.

Scarinci et al. (2013a, 2013b) proposed an extension of the normal ramp metering system using intelligent vehicles. On-ramp vehicles are released by a traffic light in coordination with gaps cyclically created on the main carriageway. A selected main carriageway vehicle for each traffic light cycle receives the direction to decrease its speed. As a consequence, a gap is created, and the upstream vehicles compact in a platoon. When the gap reaches the merging location, an on-ramp platoon is released by a green phase. Only one intelligent vehicle on the main carriageway is necessary for each such cycle, and no intelligent control is needed for the on-ramp vehicles. The control algorithm is based on macroscopic fundamental diagram and shock wave theory to identify an appropriate speed for the cooperative vehicles. Microscopic simulation results show a reduction in congestion formation and total time spent in congestion. Also, there is an increase in the proportion of vehicles able to merge before encountering the end of the merging section.

Finally, Sivaraman et al. (2013) developed a recommendation system to assist the merging into motorways. A vehicle was equipped with cameras, radar and LIDAR sensors, capable of capturing infrastructural characteristics such as lane 
and obstacle, and positions and speeds of surrounding vehicles. All the information is represented in a dynamic probabilistic drivability map showing the probability that an area is drivable. The system suggests to the driver whether the merge is recommended, and when and how to merge. The optimal acceleration is calculated using dynamic programming, and a speed range is shown to the driver. Acceleration and deceleration manoeuvres have an associated cost, and the optimal control with the lower total cost is chosen. The system practicality was evaluated over 42 merge events realised with the equipped vehicle. The recommended acceleration, steering angle, speed and whether or not the merge was recommended were recorded. No comparison with unassisted merging was provided.

\section{Overview of Merging Control Strategies}

While in the previous section a chronological review has been presented, this section gives an overview of the control strategies underlying similarities, differences and research trends. The algorithms are summarised in Table 1 based on their component characteristics, which can also be used for a convenient comparison among them. The following is a discussion for each characteristic, that is, each table row, the definitions of which were given in Section 2.

\subsection{Control Over}

Although algorithms controlling only the speed profile of merging vehicles or controlling only the gap creation on the main carriageway are present, the majority of them control both main carriageway and on-ramp vehicles. The latter type has a greater potential of improving the merging process because it can coordinate on-ramp and main carriageway movements, but this coordination requires a higher presence of intelligent vehicles and in some cases a centralised control.

\subsection{Control Direction}

Surprisingly, almost all the algorithms focus on controlling the longitudinal movement of vehicles, that is, speed profile and gap creation, and only a few use advisory or mandatory lane changes of main carriageway vehicles for facilitating the merging process (Marinescu et al., 2012; Park et al., 2011). Managing lateral movements requires more complex algorithms and control over a group of vehicles. The integration of the two types of algorithm, that is, longitudinal and lateral control, should receive more attention because, in the case of heavy traffic, using all available space, both with gap creation and lane-changing, could be the only possibility to accommodate high on-ramp flows.

\subsection{Controlled Vehicle Group}

More than half of the algorithms control an individual vehicle whilst the others expand the control over either a string or a group of vehicles. Because the ultimate aim of the control is to prevent congestion, stability should be a priority. For this reason it is desirable that future research increasingly considers the control over strings and groups of vehicles. 


\subsection{Merging Type}

Merging of a single vehicle is the scenario most often considered. The few algorithms evaluating on-ramp platoon merging make the simple assumption that a platoon behaves like a single vehicle that has the length from the front of the first vehicle to the rear of the last vehicle in the platoon (Hall \& Li, 2001; Lu et al., 2004; Scarinci et al., 2013a; Uno et al., 1999). This assumption is simplistic because a platoon of vehicles could have different dynamics requiring a smaller gap than the sum of gaps required by individual vehicles. More recent controls address the platoon dynamic in a more comprehensive way (Awal et al., 2013b; Wang et al., 2013). Facilitated merging of platoons could increase further the on-ramp demand allowed in the motorway; therefore, it should receive specific attention.

\subsection{Algorithm Type}

An interesting distinction is between centralised and decentralised algorithms. Control strategies developed for completely automated vehicles and vehicles equipped with on-board display require a central control centre for the junction. The control centre collects information, calculates the control actions and communicates with the vehicles. This centralised control requires a higher computation and communication cost than the decentralised approach. In the case of decentralised algorithms, such as the ones used by vehicles equipped with CACC, the intelligence is distributed over the vehicles involved in the decision, where each of them derives the control actions based on information exchange with vehicles within its communication range. Both approaches, centralised and decentralised, have advantages and disadvantages, so neither of them can be considered superior to the other.

\subsection{Monitor Over}

Unsurprisingly, the vast majority of the algorithms required monitoring of the main carriageway and on-ramp traffic. Thanks to the increasing availability and quality of positioning and tracking systems, and more available communication technologies, it seems that monitoring does not present a limitation for the implementation of this type of control (Vandenberghe, Vanhauwaert, Verbrugge, Moerman, \& Demeester, 2012).

\subsection{Traffic Type}

Few researchers have evaluated the algorithm performance in the case of mixed traffic (van Arem et al., 2006; Davis, 2007; Pueboobpaphan et al., 2010; Sivaraman et al., 2013; Wei et al., 2013; Xu \& Sengupta, 2003). It is unrealistic to assume a complete and instantaneous switch in the fleet to intelligent vehicles; therefore, the evaluation of the transition period is crucial. In this regard, the CACC technology could have the best chance of being implemented in the coming years, where a mixed fleet of equipped and unequipped vehicles will be travelling on motorways. 


\subsection{Intelligent Vehicle Type}

The intelligent vehicle types considered had an interesting chronological evolution. First, algorithms for completely automated vehicles requiring huge automation and a central control centre were studied. Then, algorithms for CACC-equipped vehicles, mixed traffic and a decentralised control centre were investigated, and finally algorithms for vehicles equipped with an on-board display received attention. Although the focus of this paper was not on technological aspects, it is logical to assume that completely automated vehicles require a more advanced technology than vehicles equipped with $C A C C$, and vehicles using an on-board display suggesting drivers' actions represent the most basic one. Interestingly, as is shown by the chronological review, the research studies go from the most advanced and computational demanding technology (completely automated vehicles) to less-advanced and less-demanding CACC, and lastly to the simplest on-board display.

A possible reason for this fact is that researchers started analysing the consequences at traffic flow removing entirely the human factor, that is, using completely automated vehicles. Then, established the possible improvement, the drivers have been gradually re-introduced using different control technologies, that is, CACC and on-board display. Furthermore, another possible reason is that modelling the movement and interactions of completely automated vehicles is simpler than modelling manually driven vehicles. In the first case, the vehicles move following precise trajectories. On the contrary, when human drivers are involved, more complex car-following and lane-changing models should be adopted to incorporate the complex human behaviour.

\subsection{Evaluation Method}

Finally, almost all the presented strategies are evaluated with simulation. Because an explicit representation of the merging process is required, all authors have used microscopic approaches. Few test tracks are present and so far no field test has been carried out specifically for algorithms to facilitate on-ramp merging. It is desirable that, with the benefit of a decrease in the cost of technologies, more test tracks and field tests will be performed.

\section{Overview of Evaluation Methods}

This section presents an overview of the methods used by the different authors to evaluate the algorithm performance. Table 2 summarises the method characteristics, and the following is a discussion of their similarities, differences and significant aspects. As in the previous section, the discussion is divided logically into the components used to describe the evaluation methods, that is, the Table 2 columns.

\subsection{Simulation Software}

A wide range of microscopic simulation models have been used, and a change from self-developed to commercial ones is noticeable. This is probably due to the increasing complexity of the behaviour that must be incorporated in the vehicle dynamic, and this is particularly true in the case of motorway junction modelling. In order to represent correctly the merging process and to recreate congestion, a multitude of sub-models must be incorporated: car-following, 
Table 2. Evaluation methods for merging control strategies

\begin{tabular}{|c|c|c|c|c|}
\hline Author & Simulation software & $\begin{array}{l}\text { Calibration } \\
\text { data }\end{array}$ & No. of runs & Evaluated indexes \\
\hline Yang et al. (1993) & Not stated & Not stated & Not stated & Gap and speed profiles \\
\hline Kachroo and Li (1997) & MATLAB (MathWorks, 2013) & Not stated & Not stated & Gap and speed profiles \\
\hline Antoniotti et al. (1997) & Smart-AHS (Antoniotti \& Gll, 1997) & USA & Not stated & Gap and speed profiles, queue \\
\hline Uno et al. (1999) & Not stated & Not stated & Not stated & Speed profiles \\
\hline Ran et al. (1999) & CORSIM (Halati, Lieu, \& Walker, 1997) & USA & Not stated & Average main carriageway and merging speed \\
\hline $\begin{array}{l}\text { Hall and Li (2001) } \\
\text { Kato et al. (2002) }\end{array}$ & $\begin{array}{l}\text { Not stated } \\
\text { (test track) }\end{array}$ & USA & 10 & Average waiting time in merging queue, average merging queue length \\
\hline $\begin{array}{l}\mathrm{Xu} \text { and Sengupta (2003) } \\
\mathrm{Lu} \text { et al. (2004) }\end{array}$ & $\begin{array}{l}\text { MATLAB (MathWorks, 2013) } \\
\text { (test track) }\end{array}$ & USA & Not stated & Speed and acceleration profiles, average speed and maximum braking \\
\hline van Arem et al. (2006) & $\begin{array}{l}\text { MIXIC (van Arem, Vos, \& } \\
\text { Vanderschuren, 1997) }\end{array}$ & Dutch & 5 & Number of shock waves, average speed and throughput \\
\hline Wang et al. (2007) & IDM (Treiber, Hennecke, \& Helbing, 2000) & Not stated & Not stated & Delay, throughput and flow \\
\hline Davis (2007) & Not stated & Not stated & Not stated & Speed profiles and throughput \\
\hline Kanavalli et al. (2008) & IDM (Treiber et al., 2000) & Not stated & Not stated & Delay, throughput and flow \\
\hline $\begin{array}{l}\text { Pueboobpaphan et al. } \\
\text { (2010) }\end{array}$ & MATLAB (MathWorks, 2013) & Not stated & Not stated & Vehicle kilometre travelled, average travel time and number of collisions \\
\hline Park et al. (2011) & VISSIM (PTV, 2013) & USA & 30 & $\begin{array}{l}\text { Vehicle miles travelled, vehicle hours travelled, average speed and } \\
\text { pollution }\end{array}$ \\
\hline Daamen et al. (2011) & FOSIM (VW, 2014) & Dutch & 17 & $\begin{array}{l}\text { Congestion duration, total travel time lost, number and length of shock } \\
\text { waves }\end{array}$ \\
\hline Milanes et al. (2011) & (test track) & & & \\
\hline Marinescu et al. (2012) & VISSIM (PTV, 2013) & Not stated & Not stated & Throughput and total delay \\
\hline $\begin{array}{l}\text { Scarinci et al. (2013a, } \\
\text { 2013b) }\end{array}$ & VISSIM (PTV, 2013) & Not stated & 30 & $\begin{array}{l}\text { Occurrence of congestion, late merging vehicles and time spent in } \\
\text { congestion }\end{array}$ \\
\hline Wang et al. (2013) & Simulink, MATLAB (MathWorks, 2013) & Not stated & Not stated & Speed profiles \\
\hline Awal et al. (2013b) & $\begin{array}{l}\text { kFTM (Awal, Kulik \& Ramamohanrao, } \\
\text { 2013a) }\end{array}$ & Not stated & Not stated & $\begin{array}{l}\text { Merging time, waiting time, energy consumption, flow and average } \\
\text { speed }\end{array}$ \\
\hline Sivaraman et al. (2013) & (test track) & & & \\
\hline Wei et al. (2013) & Not stated & Not stated & 10000 & $\begin{array}{l}\text { Costs associated with desired distance, accelerations, breaking distance } \\
\text { and fuel consumption }\end{array}$ \\
\hline
\end{tabular}


mandatory and discretional lane-changing, weaving, courtesy yielding and courtesy lane-changing. For this reason, developing and maintaining a microscopic simulation model are becoming impractical, and so commercial ones are used increasingly to evaluate control algorithms.

\subsection{Calibration Data}

Model calibration remains a difficult and time-consuming task. Lack of data relating to the merging process and the several parameters that must be calibrated for each sub-model make this task particularly complex. Nowadays, more data, especially from aerial video recording (Daamen, Loot, \& Hoogendoorn, 2010), offer new possibilities for calibration, and developments in calibration theory can facilitate the completion of this task. The entry 'calibration data' in Table 2 reports the nation where the traffic data used for calibration have been collected. It was not possible to give more details on the types of data or the calibration processes used because most authors did not report explicitly these important aspects. The algorithm evaluation is mostly based on simulation results; therefore, a proper calibration and validation of the model are essential. The lack of standard methods and procedures for these tasks is common to the entire field of traffic simulation, and in the last years, great efforts have been made to provide appropriate tools for development, test, use, calibration and validation of the models (MULTITUDE, 2014). It is important that future research will be based on common guidelines that will increase the quality of the investigations and facilitate the comparison among the different approaches.

A specific consideration should be made for models simulating a fleet composed completely by automated intelligent vehicles. In this case, the vehicle movements are representative of the automated driving algorithms, and not of the human driver behaviours. Therefore, the models can be simpler, with less variability and without the necessity of an intensive calibration process.

\subsection{Number of Runs}

Given the stochastic nature of microscopic simulation, several repetitions of the same scenario with different random seeds should be undertaken, as suggested by several guidelines (FHA, 2004; MULTITUDE, 2014). It is interesting to notice that multiple runs have been performed in most recent evaluations, showing that this practice is becoming increasingly standard. This is probably due to the increased awareness among the research communities and also to the significant rise in computational power, making possible several repetitions in an acceptable amount of time. Having multiple runs for each scenario also makes statistical tests possible to evaluate the algorithm performance against a reference scenario. Although several authors stated that the results are statistically significant, only one paper reported the $p$-values of the tests (Scarinci et al., 2013b). Multiple runs and statistical tests are always desirable with calibrated and validated stochastic microscopic simulation models.

\subsection{Evaluated Indexes}

The indexes evaluated as measures of effectiveness have an interesting evolution. Early evaluations used indexes mostly related to the assessment of the algorithm 
practicality, for example, gap and speed profiles of individual vehicles. Instead, more recent evaluations report proper traffic indexes such as throughput, average speed, delay and vehicle-kilometre-travelled. In the latest evaluations, also specific indexes of congestion formation and propagation have been introduced. These indexes, such as the number and length of shock waves, congestion duration and occurrence of congestion, should be reported because of primary importance in the evaluation of algorithms aimed to prevent the breakdown of traffic flow.

A final consideration should be made on the test tracks performed. Given the small number of equipped vehicles used in these tests, it is not possible to evaluate the traffic performance of the algorithms, but only the safety and the technological practicality. In the hope that more test tracks and field tests will be carried out in the near future, it is desirable that the number of intelligent vehicles involved will enable a proper evaluation of traffic performance, and not only an assessment of the technological practicality of the algorithm.

\section{Conclusions}

This paper presented a review of control strategies for facilitating the merging process using intelligent vehicles. From the review it was possible to identify a common structure, which was then used for presenting the different algorithms based on their characteristics. This structured overview, summarised in Table 1, was used for the algorithm comparison and identification of similarities and dissimilarities as well as research trends and possible future research directions. Similarly, the overview of the evaluation methods, presented in Table 2, showed significant aspects that further research should consider during the evaluation process of newly proposed algorithms.

Although research on this topic started in the early 1990s, constant innovations in communication technology and vehicle equipment have offered progressively increasing control possibilities. Furthermore, better understanding of traffic flow phenomena and more accurate simulation models allow a more precise development and testing of new control strategies. Therefore, this research field remains vastly unexplored. For these reasons, more than gaps in the research field, it is convenient to summarise some possible future research directions.

In the near future, a mix of equipped and non-equipped vehicles will travel on motorways, and in the case of V2I communication, not all the infrastructure will be equipped. Thus, it is interesting to assess the effects of each control policy during this transition period and to clarify its limitations. This can be done by evaluating different penetration rates and different driver compliance. This task introduces a higher complexity, but it is necessary for understanding the practicality of new control policies during the crucial transition phase.

CACC appears to be a particularly promising technology. Its use can be beneficial for several aspects such as string stability and proactive safety in addition to facilitating the merging process. This technology is likely to be available in standard vehicles in the next years; so, its potential use should be investigated in depth.

A further possible direction of research is the integration of longitudinal and lateral control. Integration of these two control directions could lead to a more effective use of the space available for merging. Clearly, safety issues should be 
addressed, since the delicate lane-changing process will become a part of the control loop.

The study of control policies managing a group of vehicles instead of a single vehicle should be a priority. This type of algorithm could improve string stability and allow preventive actions to avoid undesired situations using a proactive approach. Monitoring over a group of vehicles should address the problem of dealing with partial information, where some non-equipped vehicles are part of the group and do not participate in the V2V and V2I communication and control.

Finally, control policies and technologies appear ready for more comprehensive field tests and practical implementations. Tests in controlled and un-controlled environments could show traffic phenomena and drivers' reactions that do not arise during simulation studies, and so, helping to improve the control strategy design.

Based on the present review, two main points can be identified regarding the research carried out in the field of facilitated merging. The first one is the lack of standard methods for the use, calibration and validation of simulation models. The second one is the traffic indexes adopted to evaluate the traffic performance of the control strategies. It is of fundamental importance to monitor the traffic phenomena that should be avoided during the facilitated vehicle merging such as shock waves, flow breakdown and instability. Specific indexes should always be calculated in the evaluation and used to assess the quality of the control strategies. Furthermore, although particularly challenging, indexes suitable for evaluating the safety of the control actions should also be assessed. These indexes, which are not currently in common use, should be able to capture the probability of accidents and other psychological aspects related to driver behaviour, such as stress and discomfort during the merging manoeuvre. Both these critical points could be addressed by future research using standards and guidelines becoming more and more available (FHA, 2004; MULTITUDE, 2014).

The scope of the present review was specific to algorithms controlling motorway on-ramp merging using intelligent vehicles, and several related aspects, such as string stability, weaving and off-ramp manoeuvres, were not considered. An interesting extension of the present work will be to expand the review at these associated research topics and to evaluate overlaps, dissimilarities and possible integration.

\section{Funding}

This work was supported by the European Commission under [grant number EC FP7 224272] NEARCTIS project.

\section{References}

Antoniotti, M., Desphande, A., \& Girault, A. (1997). Microsimulation analysis of multiple merge junctions under autonomous AHS operation. IEEE conference on intelligent transportation system ITSC, Boston, MA, USA (pp. 147-152).

Antoniotti, M., \& Gll, A. (1997). SHIFT and SMART-AHS: A language for hybrid system engineering modeling and simulation. Proceedings of the conference on domain-specific languages, Santa Barbara, CA, Vol. 97 (pp. 14-14).

van Arem, B., van Driel, C. J. G., \& Visser, R. (2006). The impact of cooperative adaptive cruise control on traffic-flow characteristics. IEEE Transactions on Intelligent Transportation Systems, 7, 429-436. 
van Arem, B., Vos, A. D., \& Vanderschuren, M. J. (1997). The microscopic traffic simulation model MIXIC 1.3. Delft: INRO Centre for Infrastructure, Transport and Regional Development.

Athans, M. (1969). A unified approach to the vehicle-merging problem. Transportation Research, 3, 123-133.

Awal, T., Kulik, L., \& Ramamohanrao, K. (2013a). A k-leader fuel-efficient traffic model. IEEE intelligent vehicles symposium, Gold Coast, QLD, Queensland, Australia (pp. 1014-1021).

Awal, T., Kulik, L., \& Ramamohanrao, K. (2013b). Optimal traffic merging strategy for communication- and sensor-enabled vehicles. IEEE conference on intelligent transportation systems ITSC, The Hague, The Netherlands (pp. 1468-1474).

Bertini, R. L., \& Malik, S. (2004). Observed dynamic traffic features on freeway section with merges and diverges. Transportation Research Record, 1867, 25-35.

Bishop, J. R., \& Stevens, W. (1993). Precursor systems analyses of an automated highway system. 43rd IEEE vehicular technology conference, Secaucus, New Jersey, USA (pp. 364-367).

Daamen, W., van Arem, B., \& Bouma, I. (2011). Microscopic dynamic traffic management: Simulation of two typical situations. 14th IEEE international intelligent transportation systems conference, ITSC, Washington, DC, USA (pp. 1898-1903).

Daamen, W., Loot, M., \& Hoogendoorn, S. P. (2010). Empirical analysis of merging behavior at freeway on-ramp. Transportation Research Record: Journal of the Transportation Research Board, 2188, 108-118.

Darbha, S., \& Rajagopal, K. R. (1999). Intelligent cruise control systems and traffic flow stability. Transportation Research Part C: Emerging Technologies, 7, 329-352.

Davis, L. C. (2007). Effect of adaptive cruise control systems on mixed traffic flow near an on-ramp. Physica A: Statistical Mechanics and its Applications, 379, 274-290.

DfT. (2011). Design manual for roads and bridges. Great Britain: Department of Transport.

FHA. (2004). Traffic analysis toolbox. McLean, VA: U.S. Department of Transportation, Federal Highway Administration, Research, Development and Technology.

Fukutome, I., \& Moskowitz, K. (1960). Traffic behavior and on-ramp design. Highway Research Board Bulletin, 235, 38-72.

Halati, A., Lieu, H., \& Walker, S. (1997). CORSIM-corridor traffic simulation model. In R. F. Benekohal (Ed.), Traffic congestion and traffic safety in the 21st century: Challenges, innovations, and opportunities (pp. 570-576). New York, NY: American Society of Civil Engineers.

Hall, R. W., \& Li, C. (2001). Evaluation of priority rules for entrance to automated highways. ITS Journal, 6, 175-193.

Harwood, D. W., \& Mason, J. M. (1993). Ramp/mainline speed relationships and design considerations. Transportation Research Record, 1385, 121-125.

Hunter, M., Machemehl, R., \& Tsyganov, A. (2001). Operational evaluation of freeway ramp design. Transportation Research Record: Journal of the Transportation Research Board, 1751, 90-100.

Kachroo, P., \& Li, Z. (1997). Vehicle merging control design for an automated highway system. IEEE conference on intelligent transportation systems, ITS, Boston, MA, USA (pp. 224-229).

Kanavalli, A., Ranga, L. V. U., Sathish, A. G., Shenoy, P. D., Venugopal, K. R., \& Patnaik, L. M. (2008). Proactive sliding - window strategy for merging sensor-enabled cars. 16th IEEE international conference on networks, ICON, New Delhi, India (pp. 1-5).

Kato, S., Tsugawa, S., Tokuda, K., Matsui, T., \& Fujii, H. (2002). Vehicle control algorithms for cooperative driving with automated vehicles and intervehicle communications. IEEE Transactions on Intelligent Transportation Systems, 3, 155-161.

Kerner, B. S. (2007). On-ramp metering based on three-phase traffic theory — Part II. Traffic Engineering and Control, 48, 68-75.

Leclercq, L., Laval, J. A., \& Chiabaut, N. (2011). Capacity drops at merges: An endogenous model. Transportation Research Part B: Methodological, 45, 1302-1313.

Lu, X-.Y., Tan, H-.S., Shladover, S., \& Hedrick, J. K. (2001). Nonlinear longitudinal controller implementation and comparison for automated cars. Journal of Dynamic Systems, Measurement, and Control, 123, $161-167$.

Lu, X-.Y., Tan, H-.S., Shladover, S. E., \& Hedrick, J. K. (2004). Automated vehicle merging maneuver implementation for AHS. Vehicle System Dynamics, 41, 85-107.

Mammano, F. J., \& Bishop, J. R. (1992). Status of IVHS technical developments in the United States. 42nd IEEE vehicular technology conference, Denver, CO, USA (pp. 85-88).

Marinescu, D., Curn, J., Bouroche, M., \& Cahill, V. (2012). On-ramp traffic merging using cooperative intelligent vehicles: A slot-based approach. 15th International IEEE conference on intelligent transportation systems, ITSC, Anchorage, AK, USA (pp. 900-906). 
Marinescu, D., Curn, J., Slot, M., Bouroche, M., \& Cahill, V. (2010). An active approach to guaranteed arrival times based on traffic shaping. 13th International IEEE conference on intelligent transportation systems, ITSC, Funchal, Portugal (pp. 1711-1717).

Masher, D. P. (1975). Guidelines for design and operation of ramp control systems. Menlo Park, CA: Stanford Research Institute.

MathWorks. (2013). MATLAB. Retrieved from www.mathworks.com

Milanes, V., Godoy, J., Villagra, J., \& Perez, J. (2011). Automated on-ramp merging system for congested traffic situations. IEEE Transactions on Intelligent Transportation Systems, 12, 500-508.

MULTITUDE. (2014). Traffic simulation: Case for guidelines. Publications Office of the European Union, MULTITUDE project - methods and tools for supporting the Use caLibration and validaTIon of Traffic simUlation moDEls.

NEARCTIS. (2009). Preliminary report on the review of the state of knowledge and capability in relevant technologies. Technical Report. NEARCTIS Consortium, European Commission.

Papageorgiou, M., Hadj-Salem, H., \& Blosseville, J. M. (1991). ALINEA: A local feedback control law for on-ramp metering. Transportation Research Record, 1320, 58-64.

Papageorgiou, M., \& Kotsialos, A. (2002). Freeway ramp metering: An overview. IEEE Transactions on Intelligent Transportation Systems, 3, 271-281.

Park, H., Bhamidipati, C. S., \& Smith, B. L. (2011). Development and evaluation of enhanced intellidrive-enabled lane changing advisory algorithm to address freeway merge conflict. Transportation Research Record: Journal of the Transportation Research Board, 2243, 146-157.

PTV. (2013). VISSIM. Retrieved from http://vision-traffic.ptvgroup.com

Pueboobpaphan, R., Liu, F., \& van Arem, B. (2010). The impacts of a communication based merging assistant on traffic flows of manual and equipped vehicles at an on-ramp using traffic flow simulation. 13th International IEEE conference on intelligent transportation systems, ITSC, Funchal, Portugal (pp. 14681473).

Ran, B., Leight, S., \& Chang, B. (1999). A microscopic simulation model for merging control on a dedicated-lane automated highway system. Transportation Research Part C: Emerging Technologies, 7, 369-388.

Sarachik, P. E., \& Chu, K-.C. (1975). Real-time merging of high-speed vehicular strings. Transportation Science, 9, 122-138.

Scarinci, R., Hegyi, A., \& Heydecker, B. G. (2013a). Cooperative ramp metering - a study of the practicality of a ramp metering development using intelligent vehicles. 45th Annual UTSG universities' transport study group conference, Oxford, UK.

Scarinci, R., Heydecker, B. G., \& Hegyi, A. (2013b). Analysis of traffic performance of a ramp metering strategy using cooperative vehicles. 16th International IEEE Conference on Intelligent Transportation Systems - ITSC, The Hague, The Netherlands.

Shladover, S. E. (1980). Operation of merge junctions in a dynamically entrained automated guideway transit system. Transportation Research Part A: General, 14, 85-112.

Shladover, S. E. (1991). Longitudinal control of automotive vehicles in close-formation platoons. Journal of Dynamic Systems, Measurement, and Control, 113, 231-241.

Sivaraman, S., Trivedi, M. M., Tippelhofer, M., \& Shannon, T. (2013). Merge recommendations for driver assistance: A cross-modal, cost-sensitive approach. IEEE intelligent vehicles symposium, Gold Coast, QLD, Queensland, Australia (pp. 411-416).

Swaroop, D., \& Hedrick, J. K. (1999). Constant spacing strategies for platooning in automated highway systems. Journal of Dynamic Systems, Measurement, and Control, 121, 462-470.

Tignor, S. C. (1975). Operational analyses of freeway moving-merge systems. Transportation Research Record, 533, 1-21.

Treiber, M., Hennecke, A., \& Helbing, D. (2000). Congested traffic states in empirical observations and microscopic simulations. Physical Review E, 62, 1805-1824.

Uno, A., Sakaguchi, T., \& Tsugawa, S. (1999). Merging control algorithm based on inter-vehicle communication. IEEE international conference on intelligent transportation systems, Tokyo, Japan (pp. 783787).

Vandenberghe, W., Vanhauwaert, E., Verbrugge, S., Moerman, I., \& Demeester, P. (2012). Feasibility of expanding traffic monitoring systems with floating car data technology. IET Intelligent Transport Systems, 6, 347-354.

VanderWerf, J., Shladover, S., Kourjanskaia, N., Miller, M., \& Krishnan, H. (2001). Modeling effects of driver control assistance systems on traffic. Transportation Research Record, 1748, 167-174.

Varaiya, P. (1993). Smart cars on smart roads: Problems of control. IEEE Transactions on Automatic Control, 38, 195-207. 
VW. (2014). FOSIM, Freeway Operations SIMulation. Retrieved from www.fosim.nl

Wang, Y., Wenjuan, E., Tang, W., Tian, D., Lu, G., \& Yu, G. (2013). Automated on-ramp merging control algorithm based on internet-connected vehicles. IET Intelligent Transport Systems, 7, 371-379.

Wang, Z., Kulik, L., \& Ramamohanarao, K. (2007). Proactive traffic merging strategies for sensor-enabled cars. VANET'07: Fourth ACM international workshop on vehicular ad hoc networks, Montreal, QC, Canada (pp. 39-48).

Wei, J., Dolan, J. M., \& Litkouhi, B. (2013). Autonomous vehicle social behavior for highway entrance ramp management. IEEE intelligent vehicles symposium, Gold Coast, QLD, Queensland, Australia (pp. 201-207).

Xu, Q., \& Sengupta, R. (2003). Simulation, analysis, and comparison of ACC and CACC in highway merging control. IEEE intelligent vehicles symposium, Gold Coast, QLD, Queensland, Australia (pp. 237242).

Yang, C., \& Kurami, K. (1993). Longitudinal guidance and control for the entry of vehicles onto automated highways. 32nd IEEE conference on decision and control, San Antonio, TX, USA (p. 1891-1896).

Yang, C., Milacic, M., \& Kurami, K. (1993). A longitudinal control concept for merging of automated vehicles. Intelligent vehicles symposium, Tokyo, Japan (pp. 408-413).

Yi, H., \& Mulinazzi, T. E. (2007). Urban freeway on-ramps: Invasive influences on main-line operations. Transportation Research Record, 2023, 112-119. 